\title{
Corrigendum
}

\section{Variation in the Neisseria meningitidis FadL-like protein: an evolutionary model for a relatively low- abundance surface antigen}

Daniel Yero, Caroline Vipond, Yanet Climent, Gretel Sardiñas, lan M. Feavers and Rolando Pajón

Microbiology (2010), 156, part 12, 3596-3608

The shading patterns in Fig. 2(a) of the published version of this paper (page 3601) were not correctly reproduced. The correct version of the figure is shown below.

(a)

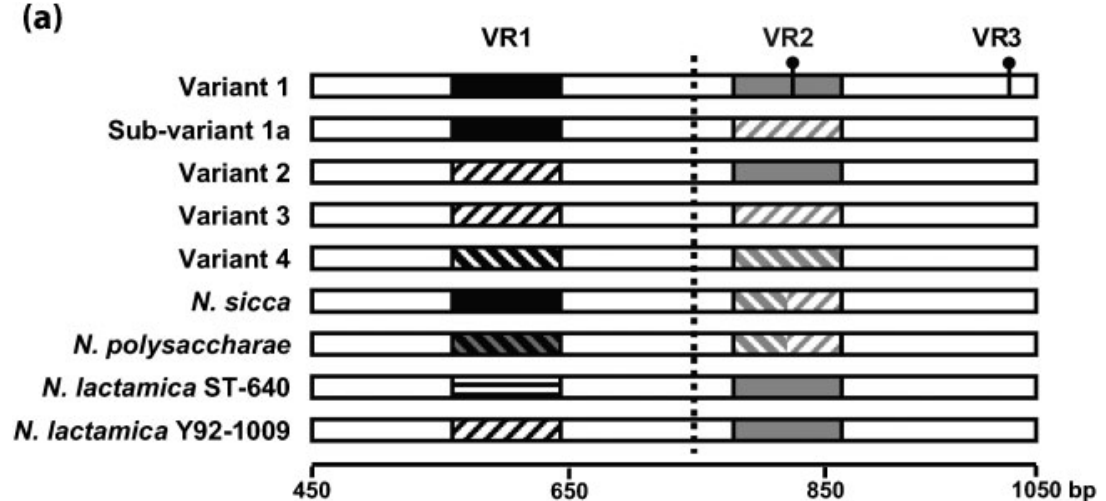

(b)

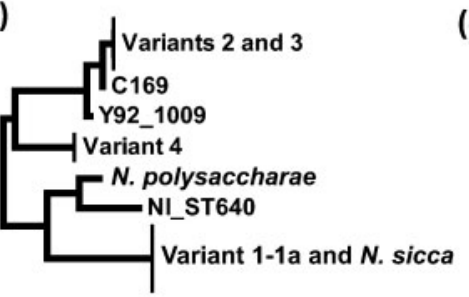

$\stackrel{0.1}{1}$ (c)

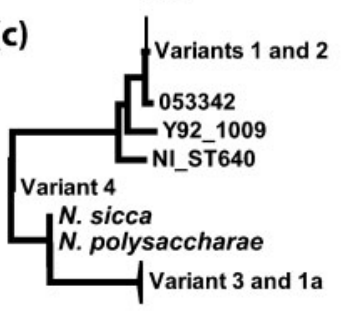

荡

Fig. 2. (a) Diagram illustrating the variability of VR1 and VR2 coding regions in fadL sequences from the four variants, subvariant $1 \mathrm{a}$ and the four commensals. Fragments with sequence identity higher than $85 \%$ are indicated with the same pattern. The potential breakpoint for recombination (position 765) is indicated by a vertical line. The positions of two sites with high evidence of positive selection are indicated by circles. (b, c) Neighbour-joining trees constructed from the aligned nucleotide sequences encoding loops 3 (VR1) (b) and 4 (VR2) (c) of the FadL protein. 